\title{
An integrated QAP-based approach to visualize patterns of gene expression similarity
}

\author{
Mario Inostroza-Ponta, Alexandre Mendes, \\ Regina Berretta, and Pablo Moscato \\ Centre for Bioinformatics, \\ Biomarker Discovery and Information-based Medicine \\ The University of Newcastle \\ Callaghan, NSW, 2308, Australia. \\ Pablo.Moscato@newcastle.edu.au
}

\begin{abstract}
This paper illustrates how the Quadratic Assignment Problem (QAP) is used as a mathematical model that helps to produce a visualization of microarray data, based on the relationships between the objects (genes or samples). The visualization method can also incorporate the result of a clustering algorithm to facilitate the process of data analysis. Specifically, we show the integration with a graph-based clustering algorithm that outperforms the results against other benchmarks, namely $k$-means and self-organizing maps. Even though the application uses gene expression data, the method is general and only requires a similarity function being defined between pairs of objects. The microarray dataset is based on the budding yeast ( $S$. cerevisiae). It is composed of 79 samples taken from different experiments and 2,467 genes. The proposed method delivers an automatically generated visualization of the microarray dataset based on the integration of the relationships coming from similarity measures, a clustering result and a graph structure.
\end{abstract}

\section{Introduction}

The analysis of gene expression data coming from microarray technologies has become an important challenge for computer scientists working in bioinformatics. Among the techniques available, the visualization of microarray data is crucial to assist the analysis. Currently, it is mainly carried out by the use of heat maps of the gene expression, which give the user a clear appreciation of how a set of genes are expressed along a set of samples, experiments or conditions. Another approach is the use of graph visualization algorithms. After modeling the microarray data as a graph (which is generally obtained with different ad hoc procedures), different graph layout algorithms are applied, like the force-direct [1] or the circular layout $[2,1]$. An important step in these visualization methods is the definition of the graph, since the result of the graph layout algorithms will depend on the structure of the graph, which in general disregard any other information from the dataset. For example, a generally employed method to build such a graph is to define a distance or similarity between genes and then 
a threshold value such that a graph will be constructed with a one-to-one map between vertices and genes; an edge connects a pair of genes if, and only if, they are at a distance smaller than the threshold value. A force-direct layout algorithm can subsequently be applied with forces that attract vertices connected by these edges, and in some cases the pairs of vertices not connected tend to be separated via ad hoc repulsion forces. As a result, it is expected that the layout of the graph will have the elements with similar expression pattern linked by an edge, and consequently closer in the layout.

In this work we propose a different visualization method for microarray data based on the modeling of the layout problem as an instance of the Quadratic Assignment Problem (QAP). Briefly, the QAP considers a set of objects to be assigned on a set of available locations, considering the flow between all the objects and the distance between all the locations, aiming to minimize the overall flow cost. The layout solution will provide a visualization of the objects (representing genes or samples) where the position of each will depend on the relationships between them in the dataset. Moreover, we combine our visualization technique with a graph-based clustering algorithm that uses a combination of $k$ Nearest Neighbors and Minimum Spanning Tree, showing the versatility of our visualization method to display the components with the integration of several levels of information. In order to illustrate the method's performance, we considered a dataset used by Eisen et al. [3] composed of the expression of 2,467 genes over 79 samples from time courses during different experiments on the budding yeast $S$. cerevisiae. We use the visualization method proposed on both genes and samples. The output shows the ability of the method to produce a layout based on the integration of different levels of information: a) similarity between objects (genes or samples), b) a representative graph structure and c) a clustering result. Also, because of the formulation of the problem, we can mathematically guarantee that all the relationships will be considered in the generation of the layout.

The paper is organized as follows: first we present the mathematical formulation of the layout problem as an instance of the QAP. Next, the graph-based clustering method is described. Furthermore, we show the computational results on the microarray dataset and the comparison with two other clustering algorithms: $k$-Means [4] and self organizing maps (SOM) [5]. Finally, the analysis and conclusions are drawn.

\section{QAP-based layout method}

The Quadratic Assignment Problem (QAP) belongs to the NP-hard class and it is a well-studied combinatorial optimization problem [6-8]. In this problem, the task is to assign a set of $n$ objects to $m$ locations $(m \geq n)$. A matrix $L=\left\{l_{i j}\right\}$ of distances between the $m$ locations is given as input, as it is also given a matrix $F=\left\{f_{i j}\right\}$ of flows between the $n$ objects. The objective is to minimize the overall transportation cost between all the objects considering both the flow between each pair of them and the distances between the locations in which 
objects are being assigned. Formally, the objective function to be minimized is the following:

$$
\operatorname{Cost}(S)=\sum_{i=1}^{n} \sum_{j=1}^{n} f_{i j} l_{S(i) S(j)},
$$

where $S(i)$ represents the assigned location of the object $i$ in solution $S$. In this paper, we are using a mathematical formalization of the visualization problem in which we have QAP instances with $n$ objects and $m>>n$ available locations. In order to create one of these instances we use the following procedure:

1. each of the $n$ objects represents either a sample or a gene;

2. a matrix $D$ of distances between each pair of objects is computed using a distance metric, for example based on Pearson Correlation, Euclidean or Cosine distances, among others;

3. the flow $f_{i j}$ (matrix $F$ ) between any pair of objects $i$ and $j$ is defined as $f_{i j}=\frac{1}{d_{i j}}(\forall i \neq j, 0$ otherwise $)$;

4. a grid of $m$ locations $(m>>n)$ is defined;

5. the distance between each pair of locations in the grid (matrix $L$ ) is calculated using Euclidean distance.

Clearly, higher flow values will be assigned to objects that are very similar and lower flow values to samples that are dissimilar. A good solution for the QAP will put the objects with a high flow closer in the layout, which is exactly our goal.

\subsection{Proximity graph clustering method}

As we mentioned in the introduction, there are several alternatives to define a graph that represents the most important proximity relationships in the dataset. Our ad-hoc proximity graph is built using information from a minimum spanning tree $\left(G_{M S T}\right)$ and a $k$-nearest neighbors $\left(G_{k N N}\right)$ graphs. A minimum spanning tree is a connected, acyclic subgraph $G_{M S T}\left(V, E_{M S T}\right)$ containing all the nodes of $G$ and whose edges total sum has minimum weight. On the other hand, a $k$ nearest neighbors correspond to the graph $G_{k N N}\left(V, E_{k N N}\right)$, where $e_{i j} \in E_{k N N}$ iff $j$ is one of the $k$ nearest neighbors of $i$.

Initially, we create a complete undirected weighted graph $G(V, E, w)$ using the distance matrix $D$, such that the weight $w_{i j}=d_{i j}$. We define our proximity graph, namely $G_{\text {cluster }}\left(V, E_{\text {cluster }}\right)$, such that $E_{\text {cluster }}=E_{M S T} \cap E_{k N N}$. This type of proximity graph was also used in González-Barrios and Quiroz (2003) [9]. In their paper, the authors fixed $k=\lceil\ln (n)\rceil$ as the parameter for the $k \mathrm{NN}$ graph, where $n$ represents the number of vertices in the graph $G$. After many tests, we adopted a variant of that expression for the value of $k$, which is shown in expression 2.

$$
k=\min \left\{\lceil\ln (n)\rceil ; \min k / G_{k N N} \text { is connected }\right\}
$$


The graph $G_{\text {cluster has } c} \geq 1$ disconnected subgraphs $\left(G_{\text {cluster }}^{1}, \ldots, G_{\text {cluster }}^{c}\right)$. The process is applied recursively on each subgraph of $G_{\text {cluster }}$ until no further partition is found. In Figure 1 we show the algorithm of this process.

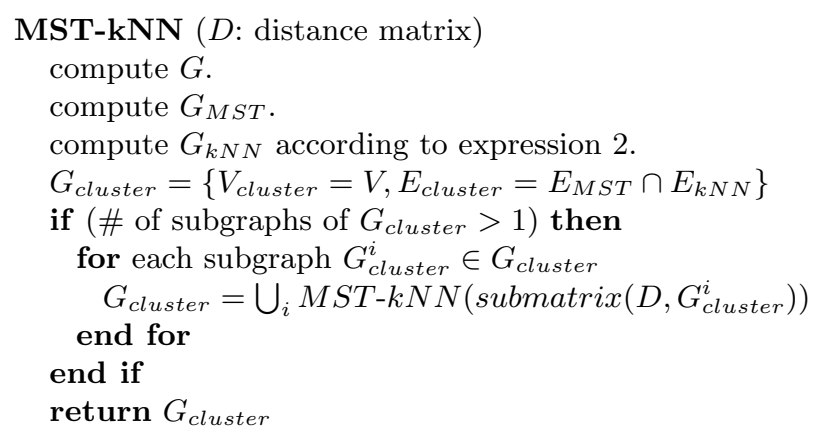

Fig. 1. Pseudo-code of the proximity graph based clustering method.

The final output is a partition of the set of elements based only on the data provided (the distance matrix $D$ between the elements) and with no userdetermined parameter. However, it is also possible to provide a maximum value for $k$ and thus have more control over the algorithm. In addition to the clusters themselves, the edges of the graph $G_{\text {cluster }}$ represent elements that are close to each other.

\subsection{Integration of proximity graph clustering with the visualization method.}

In order to integrate the result of the graph-based clustering presented in 2.1 with the visualization method proposed, we consider each subgraph $G_{\text {cluster }}^{i} \in G_{\text {cluster }}$ as a QAP instance that it will be independently optimised. In addition, for each of them, we also redefine the flow for each pair of samples (or genes) in the cluster as show in 3 , since the edges that belong to the $G_{\text {cluster }}$ are responsible for keeping the cluster together. After several tests, we define 1000 as the multiplicative factor for those edges, because it allows the method to better represent the graph structure on the layout.

$$
f_{i j}= \begin{cases}\frac{1000}{d_{i j}} & , \text { if } e_{i j} \in E_{\text {cluster }} \\ \frac{1}{d_{i j}} & , \text { otherwise }\end{cases}
$$

Following the creation of several QAP instances, one for each cluster, we have to place all clusters in a single layout. To do so, another QAP instance is created. In this case, each object represents one of the clusters $G_{\text {cluster }}^{i}$. This instance is created by building a fully connected graph $G_{C}\left(V_{C}, E_{C}, w_{C}\right)$ where $\left|V_{C}\right|=c$ 
(number of subgraphs in $G_{\text {cluster }}$ ) and the weight $w_{C_{i j}}$ corresponds to the average flow between the subgraphs $G_{\text {cluster }}^{i}$ and $G_{\text {cluster }}^{j}$, calculated as:

$$
w_{C_{i j}}=\frac{\sum_{p \in G_{\text {cluster }}^{i}} \sum_{q \in G_{\text {cluster }}^{j}} d_{p q}}{\left|V_{\text {cluster }}^{i}\right| *\left|V_{\text {cluster }}^{j}\right|}
$$

From $G_{C}$, the new QAP instance is created as was describe in section 2. To tackle the QAP problem, we use a memetic algorithm described in [10]. The memetic algorithm will produce a solution for the QAP, which will correspond to the layout of the data. The method produces a layout in two stages: first, the samples (or genes) are located according to the relationships between all the components of each cluster and finally, the position of each cluster is determined by the relationships between different clusters, considering the average distances among all components of each pair of clusters.

\section{Experiments}

\subsection{Data description}

The dataset used in this work originates from the yeast (Saccharomyces cerevisiae) gene expression microarray used by Eisen et al. [3]. The original yeast whole-genome data contains the cDNA sequences associated to 9,800 ORFs (open reading frames) and 79 samples. The samples are divided into eight reference groups associated to experiments on the budding yeast S. cerevisiae, namely alpha factor - 18 samples (alpha factor arrest and release); CDC15 15 samples (cdc15 arrest and release); cold shock - 4 samples; diauxic shift 7 samples; DTT shock - 4 samples; elutriation - 14 samples; heat shock - 6 samples; and sporulation - 11 samples. We use the freely available data (from http://www.pnas.org/cgi/content/full/95/25/14863/DC1) corresponding to the Fig. 2 of the work presented by Eisen et al. [3]. It contains a subset of 2,467 genes with functional annotations.

\subsection{Computational experiments}

The experiments were performed on samples and genes separately. Firstly, we calculate the distance matrix $D$, using as distance metric the Pearson Correlation as shown in 5 , where $\rho_{i j}$ represents the Pearson correlation either between a pair of genes or a pair of samples. This produces a distance matrix with values between 0 (if the elements are perfectly correlated) and 2 (if the elements are perfectly anti-correlated).

$$
d_{i j}=1-\rho_{i j} .
$$

To find a solution to the QAP instances, we used the Memetic Algorithm described in [10]. The methods were coded in Java, and run in a Pentium IV $(2,3$ $\mathrm{GHz}$ ) workstation. The program generates a GML file with the location of each 
sample in a grid. To visualize the file, we use the yEd Graph Editor, freely

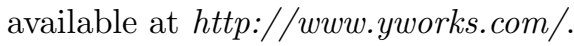

In the case of samples, since they are from eight different experiments on yeast, it is supposed that samples belonging to the same experiment, should have similar expression patterns, consequently they should be put closer in the final layout. We performed two tests: first, we applied the visualization method on the complete dataset without the use of the graph-based clustering method, to illustrate its ability to produce a layout with the samples with similar expression patterns together, and second, we combined the output of the graph-based clustering algorithm presented in 2.1 with the visualization method.

In the case of genes, we expect that genes with similar functional annotation have a similar expression profile, so, they should be assigned closer in the visualization. We present the result for the 2,467 genes in the dataset using the complete method proposed (visualization + clustering) to show how it performs in a larger dataset. We show how the method is capable of place closer genes with similar functional annotation even if they are in different clusters.

Results: In the first experiment, we applied the visualization method on the 79 samples, without the use of any extra parameter. In Figure 2 we show the layout produced by the memetic algorithm. The samples have different shapes to indicate the experiment to which they belong. It is clear in the figure that

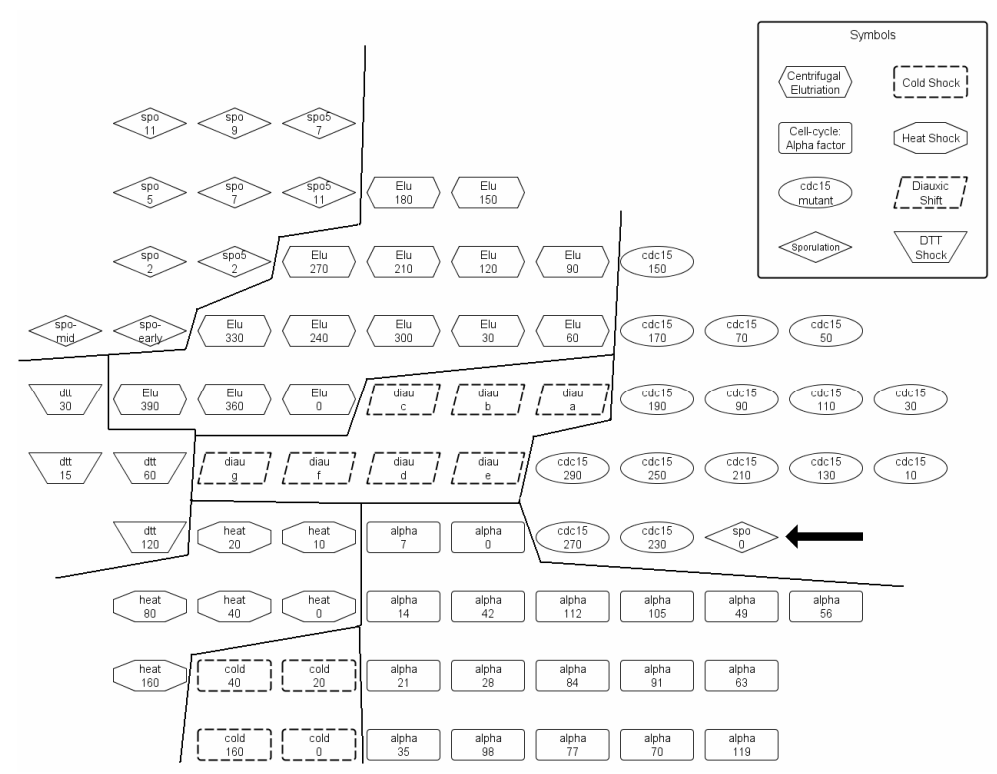

Fig. 2. QAP-based visualization of the 79 samples of yeast, considering the expression similarity between the samples. 
the organization of the samples shows elements with a similar gene expression pattern (with higher correlation) located closer, giving to the user an informative display of the data.

In the second experiment with samples, we integrate the visualization method with the graph-based clustering algorithm. It is important to clarify that we could integrate the result of any clustering algorithm with the visualization method. We first compared the solution of the graph-based clustering algorithm against $k$-means [4] and SOM [5], using the homogeneity $(\mathbf{H})$ and separation $(\mathbf{S})$ indexes [11], which give us an idea of how similar and dissimilar are the elements into a cluster and among the clusters respectively. To run the two algorithms, we used the software Expander [11], available online at http://www.cs.tau.ac.il/ rshamir/expander/ and also to evaluate the indexes $\mathbf{H}$ and $\mathbf{S}$ for each solution. Since the algorithm implementations are non deterministic, we ran each of them 10 times for different parameters. For the $k$-means algorithm, we tested for $k=8,9$ and 10, and in the case of SOM, we used grids of sizes $2 \times 4,3 \times 3$, and $2 \times 5$. In Table 1 we show the average $\mathbf{H}$ and $\mathbf{S}$ indexes for each of the algorithms with different input parameters.

Table 1. Results for $k$-means, SOM and the MST-kNN approach in terms of homogeneity and separation. Several parameters configurations are shown for $k$-means and SOM, along with the average results for 10 runs. The MST-kNN approach had the best results in both criteria.

\begin{tabular}{l|cccc}
\hline Method & Parameter & $H_{\text {avg }}$ & $S_{\text {avg }}$ & \#clusters \\
\hline$k$-means & $k=8$ & 0.487 & 0.099 & 8 \\
& $k=9$ & 0.511 & 0.094 & 9 \\
& $k=10$ & 0.526 & 0.108 & 10 \\
\hline SOM & $2 \times 4$ & 0.589 & 0.111 & 7 and 8 \\
& $3 \times 3$ & 0.579 & 0.107 & 8 and 9 \\
& $2 \times 5$ & 0.619 & 0.116 & 9 and 10 \\
\hline MST-kNN & - & $\mathbf{0 . 6 4 2}$ & $\mathbf{0 . 0 5 7}$ & 9 \\
\hline
\end{tabular}

We also ran the fuzzy $k$-means algorithm presented in [12] and the MOCK algorithm presented by Handl and Knowles in [13], for the 79 samples. In the first case, the program provided by the author gave an "out of memory" error after more than one hour processing and the latter could not recommend a specific solution from a set of solutions found by the algorithm.

We chose the clustering algorithm presented in 2.1 because it has a better performance in comparison with the two other classical algorithms, and it also provides a graph structure that allows us to show the capacity of integrate this information in the visualization. Once the clusters are obtained, we proceed to the visualization of the clusters, applying the layout process described in section 2. The resulting graph layout using the clustering generated by the MST-kNN 
is shown in Figure 3. We emphasize that the layout algorithm arranges first the objects within each cluster according to the similarity between them, and then the position of the clusters relative to each other taking into account the similarity between their components.

From a computational cost point of view, the method took less than $30 \mathrm{sec}$ to produce the layout, including the clustering algorithm (which for this dataset takes less than one second).

The final experiment corresponds to the visualization of the 2,467 genes in the dataset, as a proof of the scalability of the method. The result is showed in Figure 4. In this case, the clustering algorithm took 27 seconds and the layout less than 10 minutes, running 30 generations of the MA for each QAP instances created (52 for clusters, plus one for the clusters layout).

\section{Discussion}

Figures 2 and 3 show that the layout correlates well with the type of experiment to which the individual samples belong to. However, there is one particular sample $($ spo- 0$)$, that seems to be close to samples of other type. In both figures sample spo-0 is closer to alpha experiments. At first sight this may indicate an error, as in general the correlation with sample type is strong, however the expression pattern of sample spo-0 is closer to alpha than sporulation experiments with an average gene expression correlation of 0.112 to alpha samples and -0.010 to sporulation samples. In Fig. 5 we show the heatmap of the gene expression of alpha and sporulation experiments. The hierarchical tree on the left also has spo-0 in the same subtree that contains all alpha samples as leaves. The most similar sporulation sample to spo-0 corresponds to spo5_2 and it is the $23^{r d}$ nearest neighbor of spo- 0 , indicating that indeed spo- 0 should not be assigned close to the other sporulation samples. On the other hand, one of the issues of clustering algorithms is that they show different degrees of sensitivity, separating in more classes than necessary (showing probable subclasses) or keeping elements from different classes together. Both situations are covered by this visualization method. In the first case, if a cluster is split the layout method manages to put them together as can be seen in Fig. 3. The clustering algorithm split the experiment cdc-15, but the layout method put them together in the result. In the second case, the layout method manages to put together samples from different experiments closer (see middle-bottom cluster in Fig. 3).

For genes, we use the functional annotation of genes to show the main characteristics of the method: when a group is split the visualization manages to put it together in the grid (Protein Degradation and Protein Synthesis) and, into a cluster, it manages to organize similar genes together (Glycolysis). This result confirms the ability of the visualization method to layout together clusters with similar components, and arrange elements within a cluster according to their similarity. The groups mentioned are the main groups found by Eisen et al. [3], but with our method, we are able to find larger groups of genes with the same functional annotation than in the original work. 

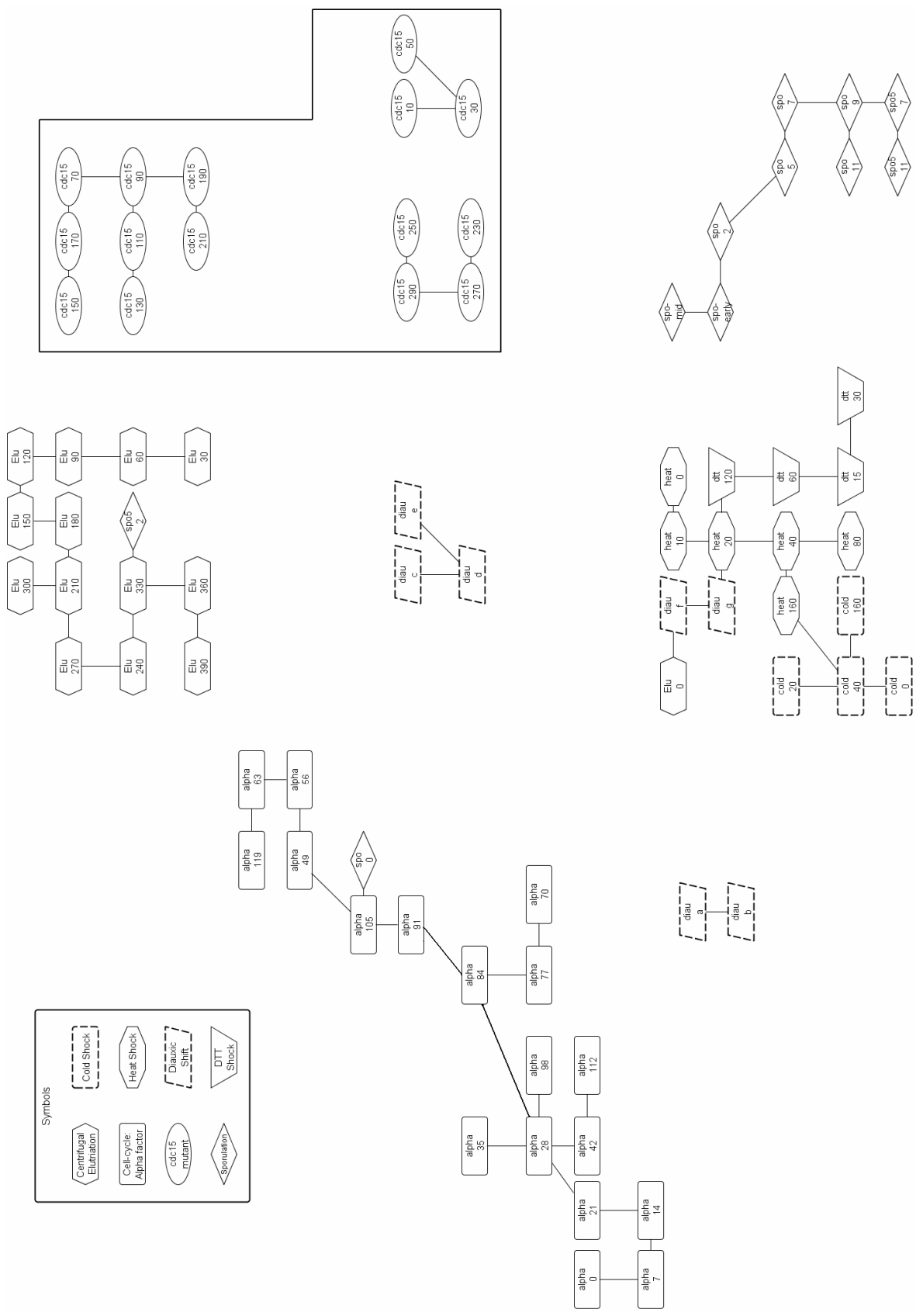

Fig. 3. QAP-based visualization of the clusters identified by the MST-kNN method. One can see that the result of the clustering method is mainly according with the experiments from which the samples come. Important, is that even when the MSTkNN approach assigns samples from the same reference group to two or more clusters, the QAP-based layout puts them in adjacent positions, e.g. cdc15 (enclosed clusters), evidencing their similarity. 


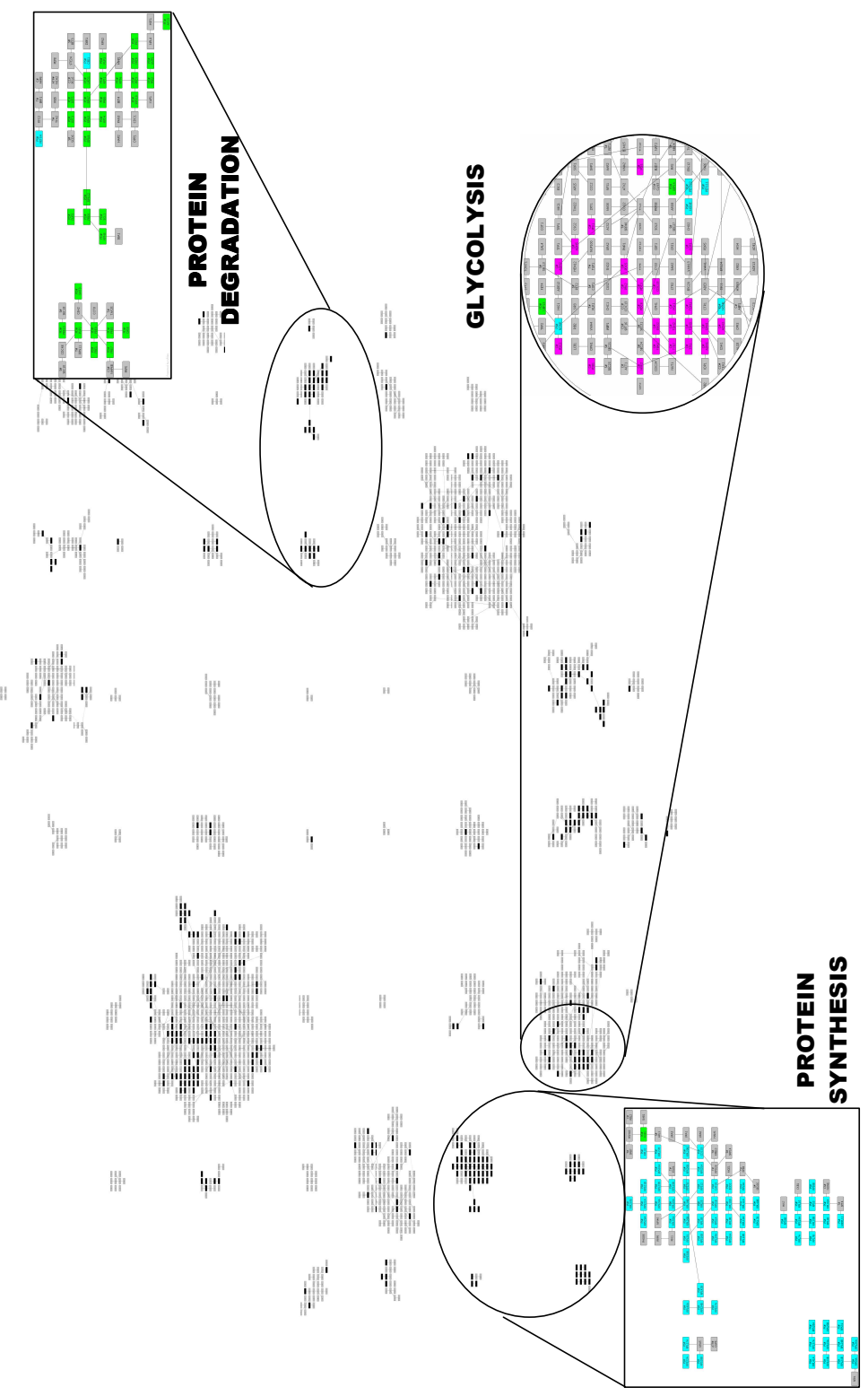

Fig. 4. Visualization of the 2,467 genes using the QAP-based approach presented. The same groups highlighted by Eisen et al in [3] are found here, but our method also put together clusters composed with a majority of genes with the same functional annotation. 


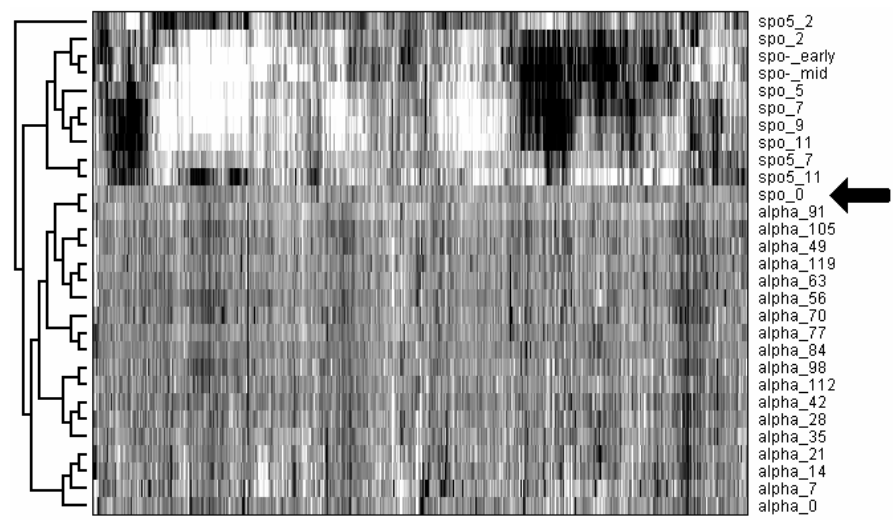

Fig. 5. Expression profiles of the samples in the sporulation and alpha factor experiments. It shows that the expression of spo0 is more similar to alpha factor samples than to other sporulation samples (see arrow).

\section{Conclusions}

We have presented a visualization method for the analysis of gene expression data coming from microarray technology. The method is based in a mathematical formulation of the visualization problem as instances of the QAP. The proposal is competitive with others and it provides a novel visualization approach. The main advantages are:

1. The position of the objects are decided according to the relationships among all the objects (either samples or genes) in the dataset, giving to the user a layout based on the whole information provided by the dataset.

2. The combinatorial optimization approach proposed here is novel, and can be synergistically "seeded" by other algorithms, like a force-directed approach allowing it to have a better starting point.

3. The ability to integrate the result of a clustering technique with the visualization method provides an easily interpretable positioning of the elements, with optimization for the layout within a cluster, and a global optimization of the layouts of the clusters.

4. Finally, the method can integrate a graph structure in the visualization by increasing the flows of some relationships.

Most of the software packages that are available for gene expression analysis lack of visualization systems that provide a reliable and information-based layout that can assist the interpretation of the datasets. Most of them consider only some graph-layout algorithms and generally they only use heatmaps. The method proposed arises as a good alternative to be integrated in the current software packages for microarray data analysis, because it has a good performance, it is scalable and its characteristics make it unique. 
Acknowledgment - This work has been supported in part by an ARC Discovery Project (DP0559755, Evolutionary algorithms for problems in functional genomics data analysis) and the ARC Centre in Bioinformatics. Mario InostrozaPonta also acknowledges the support of the Department of Informatics Engineering, University of Santiago of Chile and the Newcastle Bioinformatics Inititative.

\section{References}

1. Shannon, P., Markiel, A., Ozier, O., Baliga, N., Wang, J., Ramage, D., Amin, N., Schwikowski, B., Ideker, T.: Cytoscape: A software environment for integrated models of biomolecular interaction networks. Genome Research 13 (2003) 24982504

2. Kohler, J., Baumbach, J., Taubert, J., Specht, M., Skusa, A., Ruegg, A., Rawlings, C., Verrier, P., Philippi, S.: Graph-based analysis and visualization of experimental results with ondex. Bioinformatics 22(11) (2006) 1383-1390

3. Eisen, M., Spellman, P., Brown, P., Botstein, D.: Cluster analysis and display of genome-wide expression patterns. Proc Natl Acad Sci U S A 95 (December 1998) $14863-14868$

4. Tavazoie, S., Hughes, J., Campbell, M., Cho, R., Church, G.: Systematic determination of genetic network architecture. Nat Genet (22) (1999) 281-285

5. Tamayo, P., Slonim, D., Mesirov, J., Zhu, Q., Kitareewan, S., Dmitrovsky, E., Lander, E., Golub, T.: Interpreting patterns of gene expression with self-organizing maps: methods and application to hematopoietic differentiation. Proc Natl Acad Sci (1999)

6. Burkard, R., Çela, E., Pardalos, P., Pitsoulis, L.: The quadratic assignment problem. In Pardalos, P., Du, D., eds.: Handbook of Combinatorial Optimization. Kluwer Academic Publishers (1998) 241-338

7. Taillard, E.: Robust taboo search for the quadratic assignment problem. Parallel Computing 17(4-5) (1991) 443-455

8. Oliveira, C., Pardalos, P., Resende, M.: Grasp with path-relinking for the quadratic assignment problem. In Ribeiro, C., Martins, S., eds.: Lecture Notes in Computer Science. Volume 3059. Springer-Verlag (2004) 356-368

9. González-Barrios, J., Quiroz, A.: A clustering procedure based on the comparison between the $\mathrm{k}$ nearest neighbors graph and the minimal spanning tree. Statistics \& Probability Letters 62(1) (2003) 23-34

10. Inostroza-Ponta, M., Berretta, R., Mendes, A., Moscato, P.: An automatic graph layout procedure to visualize correlated data. In Bramer, M., ed.: Artificial Intelligence in Theory and Practice: Ifip 19th World Computer Congress. Volume 217 of IFIP International Federation for Information Processing., Springer (2006) $179-188$

11. Shamir, R., Maron-Katz, A., Tanay, A., Linhart, C., Steinfeld, I., Sharan, R., Shiloh, Y., Elkon, R.: Expander-an integrative program suite for microarray data analysis. BMC Bioinformatics 6(232) (2005)

12. Gasch, A., Eisen, M.: Exploring the conditional coregulation of yeast gene expression through fuzzy k-means clustering. Genome Biology 3(11) (2002)

13. Handl, J., Knowles, J.: Multiobjective clustering with automatic determination of the number of clusters. Technical Report TR-COMPSYSBIO-2004-02, UMIST, Manchester, UK (2004) 